\title{
Los discursos sobre tecnologías y educación en la esfera pública
}

\section{Landau, Mariana}

Resumen:

Este trabajo analiza los discursos de la prensa escrita sobre la problemática de la integración de tecnologías de la información y la comunicación (TIC) en el sistema educativo en los momentos de lanzamientos de iniciativas de políticas educativas vinculadas con la temática en la última década en la Argentina. En este caso, nos abocaremos al estudio de los titulares de la prensa escrita vinculado con la modificación de las normas que regulan el uso del celular y otros dispositivos digitales de propiedad de los estudiantes en el ámbito escolar en la provincia de Buenos Aires.

Palabras clave: Prensa escrita - TIC -

Cuadernos del Centro de Estudios de Diseño y Comunicación N ${ }^{072}$

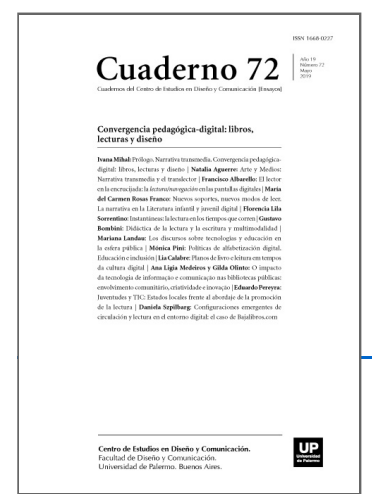

ISSN: 1668-0227

Convergencia

pedagógica-digital:

libros, lecturas y

diseño

Año XIX, Mayo 2019, Buenos Aires,

descargar PDF

ver índice de la publicación

Ver todos los libros de la publicación

compartir en Facebook

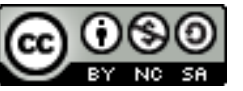

Esta obra está bajo una Licencia Creative Commons Atribución-NoComercialCompartirlgual 4.0 Internacional

Celulares - Políticas pública.

(*) Magister en Análisis del Discurso (UBA). Licenciada en Ciencias de la Educación (UBA). Profesora adjunta regular a cargo de Tecnologías educativas (Landau). Carrera de Ciencias de la Comunicación. FSOC (UBA). Profesora de la Maestría en Educación, Lenguajes y Medios de la Universidad Nacional de San Martín (UNSAM). Directora del proyecto UBACYT 20020150200214BA. Prácticas educativas mediatizadas en el discurso público en la Argentina. 2016-2018. Carrera de Ciencias de la Comunicación. Facultad de Ciencias Sociales (UBA).

Introducción

La introducción de tecnologías digitales en el sistema educativo constituye un tema de la agenda de la política educativa desde hace varias décadas. La distribución de diferentes dispositivos (los gabinetes de computación con las computadoras personales, las netbooks, las pizarras digitales, entre otras); la instalación de la infraestructura necesaria para brindar conectividad; la capacitación de los docentes; y el diseño y desarrollo de contenidos digitales han sido componentes nodales de estas iniciativas. 
En América Latina, estas políticas han enfatizado más los aspectos vinculados con el acceso, es decir, el equipamiento y en menor medida, la conectividad y la capacitación docente, que el desarrollo de estrategias de facilitación y apropiación de estas tecnologías para la enseñanza (Sunkel, Trucco y Espejo, 2014). Algunos consideran que este énfasis se debe a que constituye una primera etapa. Sin embargo, muchos de los discursos que se desplegaron en el marco de las políticas depositaron una excesiva confianza en las posibilidades de los dispositivos y en el acceso a Internet.

A partir de las políticas públicas de inclusión digital en la modalidad Uno a Uno, la visibilidad de estas iniciativas se ha incrementado. En particular, este fenómeno ha tenido una importante notoriedad en la zona del Río de la Plata (Argentina y Uruguay) por la implementación de políticas universales de acceso a las herramientas digitales.

A pesar de todo este despliegue, en términos de políticas y recursos destinados al sector, muchas investigaciones han señalado las dificultades, obstáculos y formas de apropiación diferenciada que realizan las escuelas respecto de los lineamientos enmarcados en estas políticas. En este sentido, constituye un saber ya instalado que no existe una relación directa entre la implementación de políticas de integración digital y la apropiación productiva por parte de las escuelas.

En esta investigación se parte del siguiente supuesto: la política educativa y los medios de comunicación constituyen una unidad de análisis indisociable atravesada por el proceso de mediatización. Los discursos de la esfera pública fortalecen su instalación como agenda de la política educativa a través de la presencia significativa de textos sobre la temática. Asimismo, en la última década ha habido un solapamiento entre la esfera de los medios y la representación política, de modo tal que consumir un medio -y no otro- se convirtió en símbolo de una perspectiva particular.

En particular, este trabajo analiza los discursos de la prensa escrita sobre la problemática de la integración de tecnologías de la información y la comunicación (TIC) en el sistema educativo en los momentos de lanzamientos de iniciativas de políticas educativas vinculadas con la temática en la última década en la Argentina. En este caso, nos abocaremos al estudio de los titulares de la prensa escrita vinculado con la modificación de las normas que regulan el uso del celular y otros dispositivos digitales de propiedad de los estudiantes en el ámbito escolar en la provincia de Buenos Aires.

Las TIC en el sistema educativo. Una política mediatizada

La mediatización refiere a los cambios socioculturales que atraviesan las prácticas sociales, los procesos políticos y las formas de producción de las sociedades actuales vinculados con la ubicuidad de los medios de comunicación en la vida cotidiana y con los procesos de digitalización de la información que se configuran como condición de posibilidad de las prácticas sociales, de la acción política y de la conformación de la subjetividad. La mediatización hace foco en las formas en que se articulan los medios de comunicación, las prácticas sociales y los procesos políticos, los circuitos de circulación de los discursos que en los medios se producen/reproducen y las formas de apropiación y resignificación local de esos discursos. Desde la hipótesis de la realidad manufacturada, los acontecimientos sociales se configuran en torno a su presencia y visibilidad en los medios, es decir, los medios no copian sino que "producen realidad social". El concepto de industria cultural, acuñado por la escuela de Frankfurt, es reformado para enfatizar los aspectos fabriles de la producción de la realidad social: 
"la actualidad [...] tiene el mismo status que un automóvil: es un producto, un objeto fabricado que sale de esa fábrica que el medio informativo". (Verón, 1983, p. III)

Las políticas de introducción de tecnologías en el sistema educativo están fuertemente mediatizadas. Por un lado, desde un nivel fáctico, en cada una de las instancias de lanzamiento de los programas de TIC, la esfera estatal construyó como destinatario a la prensa y a su vez la prensa configuró un discurso sobre ello. Al menos en este campo, el discurso político tiene como destinatario y construye su realidad a través de los medios de comunicación porque entre otros aspectos, a través de ellos se comunica la política. Es decir, los medios de comunicación son uno de los mediadores privilegiados de la política educativa. Por otro, desde un nivel discursivo, las políticas TIC son interpeladas por el significante tecnológico que opera a nivel imaginario como solución y respuesta a los problemas y demandas educativas (Da Porta, 2015).

Asimismo, en términos de las condiciones de producción del discurso, es necesario tomar en cuenta las características de los medios masivos de comunicación como un sector económico y como grupo de poder. En otras palabras, es necesario considerar que los medios no son sólo medios sino multimedios o industrias infocomunicacionales (Becerra y Mastrini, 2009), es decir, empresas con intereses específicos en el campo de la introducción de las tecnologías educativas y digitales como ser la provisión de Internet, diseño de contenidos, venta de software, hardware, aplicaciones, libros de texto.

En relación con la distribución como categoría semiótica, la prensa escrita constituye un espacio de referencia para los demás medios y redes sociales. Es decir que, más allá de ser la televisión el dispositivo de mayor consumo y de la emergencia de las redes sociales como espacios de circulación y producción de información, la prensa escrita aún posee un lugar destacado en la conformación de opiniones, miradas y tendencias respecto de las problemáticas sociales que aborda. Para la prensa escrita, las redes sociales son criterio de noticiabilidad, fuente de información pero también canal de comunicación con los lectores (Becerra y Beltrán, 2014). Asimismo, muchas veces los diarios on-line acarrean y traspasan el lugar logrado a través de su edición impresa.

Específicamente, sobre las TIC en el sistema educativo, el discurso de los medios como un espacio de fomento, apoyo y motorización de la política es una construcción que se configura como un dado, como un saber compartido y aceptado. Para el caso de la política uruguaya:

La llegada de la ceibalita al hogar fue acompañada de un imaginario favorable al Ceibal, intensamente promovido por los medios de comunicación, que asocia el progreso de la nación uruguaya y el incremento de las oportunidades de desarrollo social, con la distribución igualitaria, el acceso pleno y la adquisición de habilidades por parte de los niños y adolescentes para manejar la ceibalita. (Winocur y Sánchez Vilella, s/d)

Para el caso de la Argentina, esta aceptación y necesariedad de la política también estuvo presente y las diferencias fueron emergiendo con relación a la implementación. Los posicionamientos de la prensa escrita sobre Conectar Igualdad reprodujeron los enfrentamientos entre los medios y el poder ejecutivo que atravesaron los últimos años del gobierno kirchnerista (Landau, 2015).

Estas diferencias entre las formas de abordar los programas de TIC entre Argentina y Uruguay pueden ser explicadas, por un lado, por la distinta relación entre la esfera política y el campo de los medios de comunicación: mientras diferentes gobiernos coincidieron en caracterizar a los medios privados como actores 
políticos en distintos momentos de inflexión: el golpe de Estado en Venezuela en 2002, el conflicto del campo en Argentina en 2008, los enfrentamientos por la nueva Constitución en Bolivia en 2009 y el levantamiento policial en Ecuador en 2010, este conflicto en Uruguay no sucedió (Schuliaquer, 2017).

Con relación a estas diferentes formas de negociación de las escenas mediáticas en el campo educativo, en el proyecto de investigación "Mediatización y privatización en América Latina"1 se compararon las estrategias discursivas de dos grandes grupos de medios de Argentina, Chile, Ecuador y México: el primero de corte neoliberal y conservador y el segundo, con una perspectiva crítica y/o alternativa. En este marco, se considera:

El primer grupo corresponde a los diarios que tienen la mayor circulación y cantidad de lectores y además,

Son los más retomados en el discurso público, en los discursos políticos, en los discursos empresariales, en los discursos educativos. Son quienes definen las temáticas de lo que se debe discutir, de lo que se debe pensar, y de lo que se debe sentir en la actualidad, aquello que nos pasa a todos y todas. Aunque esta hegemonía hoy está matizada por los medios independientes, sigue siendo dominante su posición en la construcción de agenda. (Da Porta, 2015)

A partir del análisis de no solo los artículos de Opinión (en los que puede observarse la ideología de una forma más explícita) sino también de las Noticias y las Crónicas -ya que estos textos utilizan un estilo más "objetivo", "neutral" y distante en los que se privilegia la descripción como género discursivo- las autoras de esta investigación afirman que existe una importante correspondencia entre el posicionamiento del diario y lo que opinan los especialistas que en los medios hegemónicos están representados por los profesionales vinculados a los think tanks y en la prensa de corte progresista con intelectuales y acadé- micos de espacios universitarios y de investigación.

Los marcos en los que comunican la información los diarios es, en primer lugar, el de la crisis en general, pero también de lo educativo y de lo público. Frente a un escenario de caos que describen las noticias periodísticas, el Estado es caracterizado como inoperante. La segunda dimensión que señalan las autoras es la de una perspectiva general orientada al modelo de mercado y a la privatización. Más allá de las mitigaciones operadas por el género discursivo, es decir, por los intentos de mantener ciertos rasgos de "neutralidad", la orientación hacia la privatización se manifiesta en: la visión instrumental que articula la educación con la productividad y con la lógica mercantil; el dominio de un lenguaje técnico-economicista en el que predominan los estándares globales de evaluación de la calidad educativa; ponderación positiva de lo privado (ONG, empresas, especialistas) frente al desmanejo, la desidia y la ineficiencia de la escuela pública y de la órbita estatal (Da Porta y Cianci, 2016).

Sin embargo, las diferencias sobre la política educativa no se restringen a la esfera mediática sino que las miradas antagónicas también se hicieron presentes en la producción de conocimiento desde la esfera estatal sobre la introducción de TIC en el sistema escolar. Al respecto, Dussel (2016, p. 152) señala diferencias en las evaluaciones realizadas en Argentina y Uruguay respecto de las políticas Uno a Uno implementadas en dichos países. Así, señala la autora, mientras que en Uruguay los informes críticos se erigían en herramientas para reformular las líneas de acción; en el caso de la Argentina, en especial las agencias gubernamentales "sintieron el peso de construir legitimidad para los programas, lo que se evidencia tanto en el modo de producción de las investigaciones como en la comunicación de sus resultados" 
El análisis de los discursos de los medios de comunicación sobre educación constituye un elemento sustantivo para comprender la trama de la esfera pública. Espacios discursivos que, merced a los dispositivos electrónicos y a las nuevas configuraciones mediáticas, permiten un registro de lo que allí acontece.

Sue Thomas ha desarrollado una línea de investigación orientada a comprender las particularidades del discurso público sobre educación analizando las articulaciones entre la política educativa y los discursos de la prensa escrita. En su investigación sobre las relaciones entre la política de revisión curricular en Queensland (Australia) y los discursos de la prensa escrita muestra cómo la dinámica de la esfera pública posibilitó la construcción de un discurso público compartido sobre la política educativa, las escuelas y los docentes. En este sentido, el trabajo demuestra la capacidad de la esfera pública de definir la agenda educativa y las identidades de forma particular (Thomas, 2004). Parte de la formulación de Habermas sobre esfera pública para su reconsideración. En particular, las críticas se orientan a lo universal de la perspectiva que elude los procesos de fragmentación y pluralidad de la vida social. En este sentido, entiende a la esfera pública como un espacio de producción y circulación de discursos, como una arena de disputa y articulación, y como un espacio de configuración de identidades, entre otros aspectos.

Para analizar los discursos en la esfera pública vinculados con las políticas TIC en el sistema educativo se utilizará la perspectiva de la lingüística para comprender las estrategias discursivas utilizadas en la prensa escrita para construir/reconstruir los marcos conceptuales que atraviesan a esta dimensión de la agenda de la política educativa (Lakoff, 1995; 2007). Esta perspectiva teórica parte del supuesto según el cual las personas no eligen en función de la información que poseen sobre un tema sino de sus valores y estereotipos culturales. Los marcos de pensamiento, las metáforas y los prototipos son las estructuras a partir de las cuales comprendemos, valoramos y actuamos sobre la realidad. En un concepto complejo pueden confluir una o más metáforas y marcos de pensamiento. Muchos de estos conceptos como libertad, desarrollo, justicia son controversiales. Para el caso educativo, el concepto de "buena escuela" claramente lo es, como también lo es qué es política TIC. En educación existen dos marcos generales que permiten comprender el funcionamiento de las instituciones escolares: por un lado, el marco producción (las escuelas son fábricas, las ideas y la información son objetos sólidos, la mente de los estudiantes es un recipiente vacío, la enseñanza es transmisión de información, el aprendizaje es recepción de información); y por otro, el marco cultivo (las escuelas son jardines, las mentes y a veces el aula son el suelo, las ideas ( $y$ a veces los estudiantes) son las plantas, la enseñanza es cuidar del jardín, el aprendizaje es crecimiento (Haas y Lakoff, 2009).

Asimismo, desde esta perspectiva teórica, se han analizado los discursos políticos desde la metáfora de la "nación como familia". En esta línea es posible identificar el modelo del "padre estricto" y el modelo del "padre protector". En el primer caso, la idea de autoridad de los ejecutores de la política y el lugar de seguidores de la mayor parte de los ciudadanos se contrapone con el modelo de autoridad que se encarga de garantizar la distribución de los recursos en función de las necesidades de la población.

Estas conceptualizaciones en torno a las metáforas permitirán abordar a la prensa escrita como espacio de producción de sentido en torno a la inclusión de tecnologías que trasciende claramente el carácter instrumental que muchas veces asume esta problemática. 
De las computadoras personales a los celulares

La tecnología que ha acompañado el surgimiento y legitimidad del sistema educativo ha sido el libro escolar: libro de texto, manual y libros de lectura, entre otras. Este dispositivo que ha atravesado distintas modificaciones, en su interior tanto desde el diseño como desde el contenido, es un artefacto cultural diseñado especialmente tanto en sus contenidos como desde la forma de enunciar por el sistema escolar.

Más allá de que siempre es un texto atravesado por una trama de negociaciones y tensiones entre distintas esferas de la sociedad (Estado, asociaciones profesionales, campo académico, administración educativa, empresas e iglesias, entre otros) constituye claramente un invento escolar. A diferencia de esta tecnología, las computadoras personales no lo son. En la historia de tecnologías digitales en el sistema educativo, al menos en la Argentina, observamos básicamente tres momentos:

\section{La computadora en la escuela}

Las escuelas adquieren las computadoras personales ya sea a través de la compra por parte del Estado, de la Cooperadora escolar2 o de los dueños de las escuelas privadas. Es la escuela la encargada del mantenimiento, actualización y la responsable de la seguridad del bien adquirido. Esta computadora se incorpora y permanece siempre al interior de los muros de la institución escolar.

En términos de hardware, el prototipo de esta dimensión es el modelo de gabinete o laboratorio, es decir, la existencia de un aula con computadoras dedicadas a la enseñanza aunque también se han ensayado otros tipos de distribución en los pasillos o los carritos. En términos de software, la escuela regula las aplicaciones que se instalan en las computadoras escolares y de este modo, puede resguardar cierta homogeneidad en los programas que se utilizan. En este modelo la distancia entre las categorías "computadora escolar" y los dispositivos digitales hogareños presenta límites claros en los espacios y tiempos como también las actividades que se realizan. De este modo, se "preserva" la actividad académica del ocio (video juegos, redes sociales).

\section{La computadora va y viene. Entre la escuela y la casa}

Con la emergencia de los modelos Uno a uno, es decir, con las líneas de acción basadas en la provisión de netbooks y tabletas a cada uno de los estudiantes del sistema educativo, las computadoras ya no están únicamente en la escuela.

Quizá uno de los modelos más importantes de esta línea sea el programa One laptop per child3 (Una computadora por niño) que con base en el MIT, lanzó una propuesta de penetración en los sistemas educativos de todo el mundo y más específicamente de provisión de una computadora de bajo costo a los niños más pobres del planeta. El primer principio de este programa es que la laptop es del niño y que al finalizar la jornada escolar la computadora va al hogar para ser compartida con la familia.

En términos de software, las políticas de equipamiento llevan instalados una serie de programas educativos pero los estudiantes y sus familias pueden agregar otros. Es decir, se asegura un núcleo común de programas pero hay un nivel de variabilidad en las decisiones de cada entorno familiar, que tiene la posibilidad de incorporar aplicaciones vinculados con el curriculum, como no. 
En este modelo los límites entre lo escolar y lo "vernáculo" no son tan claros ni es deseable que lo sean. Se ponderan positivamente las sinergias, los puentes y articulaciones entre la escuela, la familia y los medios de comunicación, entre otras esferas de la sociedad. Aunque esto no sea sencillo ni fácil de lograr.

No resulta sorprendente que cuando los dispositivos son adquiridos por el centro escolar o sus inversores, el personal se muestre reacio a que se usen fuera del centro escolar, por la mayor exposición a riesgos de pérdida, deterioro u olvido del dispositivo en el hogar. Esta restricción reduce el beneficio que se obtiene de poseer unos dispositivos móviles capaces de apoyar el aprendizaje, tanto en el hogar como en el centro. Y es que cuando se limita el uso del dispositivo sólo al aula durante las clases, se restringen los beneficios a conseguir, algunos de los cuales dependen tanto del uso de los dispositivos móviles en situaciones de aprendizaje auténtico, como de la personalización y sentimiento de propiedad del dispositivo por parte de los estudiantes. (INTEF, 2016, p. 9)

3. De la casa a la escuela. "Lleva tu propio dispositivo"

En este modelo los estudiantes llevan su propia tecnología o dispositivo a la escuela4 para ser utilizados con propósitos educativos. Las razones de esta estrategia son muchas: la rápida obsolescencia de los equipos, la presencia de más de una pantalla en muchos de los hogares, la utilización casi permanente de dispositivos móviles por parte de niños y jóvenes, la capacidad de procesamiento cada vez mayor de los teléfonos celulares inteligentes y tabletas, entre otras.

Aquí, los estudiantes son los dueños de las herramientas tecnológicas. Entonces, la escuela debe regular sobre un dispositivo del que no es dueña ni ha entregado. La diversidad signa al hardware y al software ya que no todos los equipos son iguales ni en el sistema operativo, ni en los programas ni en las aplicaciones que se utilizan.

Más allá de ser una perspectiva consistente con los contextos de alta disponibilidad tecnológica es posible señalar los siguientes obstáculos en su implementación:

- La desigualdad que se materializa en el acceso diferencial a los dispositivos.

- La dificultad de generar propuestas de enseñanza homogéneas en contextos de diversidad de dispositivos.

- La seguridad de los equipos.

- La necesidad de contar con la velocidad necesaria para sostener la conexión simultánea de gran cantidad de equipos.

- El soporte técnico.

- Las diferencias en los dispositivos y su capacidad de procesamiento.

En síntesis, existen distintos modelos de equipamiento que regulan la adquisición y uso al interior de la escuela y su articulación con el contexto familiar y personal de los estudiantes y sus familias. No se trata de un modelo evolutivo en el que cada etapa que se inicia supera a la anterior sino de estrategias que operan según las 
posibilidades, objetivos y recursos existentes. En cada una de estas estrategias las escuelas debe asumir distintas posiciones.

En este trabajo nos interesa analizar el discurso de los medios con relación al discurso de la política representado por las resoluciones que regulan el uso del celular por parte de estudiantes y docentes en la provincia de Buenos Aires. Nos interesa abordar esta política porque después de 15 años en los que las políticas TIC estuvieron fuertemente asociadas a la entrega de dispositivos, a la generación de contenidos, a la capacitación, es decir, a proveer de ciertos elementos al sistema educativo, las resoluciones vinculadas a "trae tu propio dispositivo" tienen por fin regular la presencia de artefactos "extra-escolares". Es decir, reconocen la presencia de otros artefactos culturales y establecen una voz acerca de ellos.

Las metáforas de la prensa escrita sobre la política TIC vinculada con el uso del celular en la provincia de Buenos Aires

En el año 2006 la Dirección General de Cultura y Educación de la provincia de Buenos Aires dictó una normativa que establecía la prohibición de utilizar celulares por parte de docentes y alumnos. Entre otros fundamentos, se esgrimía que el uso del celular obstaculiza el aprendizaje y que existen espacios diferenciados para cada medio de comunicación: "Que para cada medio de comunicación existe un espacio propio de utilización e incoordinación funcional" (Resolución 1728/06). Es decir, establecen una clara diferenciación entre el espacio escolar y no escolar. En el año 2016, tras el cambio de gobierno, esta resolución es derogada habilitando el uso de distintos dispositivos digitales con fines pedagógicos (Resolución 778/2016).

Esta prohibición de uso de celulares en las escuelas se desarrolló en forma paralela al despliegue de una iniciativa de carácter universal de distribución de equipamiento al interior del sistema educativo para el nivel medio y superior como fue Conectar Igualdad. En este sentido, es posible señalar que las TIC son un conjunto heterogéneo de dispositivos, prácticas y recursos que adquieren valoraciones diferenciadas por parte de la sociedad y de la escuela. Así, mientras en la esfera social Internet es vista como un espacio público y abierto, el celular es asociado a las redes privadas, personales y locales (Winocur, 2009), la escuela diferencia qué dispositivos y usos son "pedagógicos" de los que no lo son.

Estas valoraciones se encuentran enraizada en prácticas y representaciones sociales que establecen formas deseables y usos apropiados y contextualizados de estas tecnologías. Este imaginario se encuentra atravesado por los discursos de los medios de comunicación que median, entre otros aspectos a través de las metáforas que utilizan, en la comprensión de la esfera pública. Para analizar los discursos vinculados con las TIC, en esta oportunidad hemos seleccionado las metáforas de los titulares de la prensa escrita vinculados con la normativa relacionada con el uso del celular y otros dispositivos digitales de uso doméstico en los diarios argentinos de alcance nacional.

Las metáforas no constituyen únicamente un recurso estilístico del lenguaje sino que son un elemento estructural del sistema conceptual. Implican experimentar una cosa en términos de otra, es decir, valerse de otro dominio para pensar un objeto.

A continuación se presentan dichos titulares: 
La Nación

- Levantan la prohibición de usar celulares en las aulas bonaerenses5

Lo decidieron las autoridades provinciales; los alumnos podrán llevar sus propios teléfonos, tablets y notebooks para emplearlos como parte del proceso pedagógico

- Autorizan el uso de teléfonos celulares en las escuelas bonaerenses6

Se podrán usar para cuestiones pedagógicas; derogaron la resolución que lo impedía desde 20067

Clarín

- Fin del "cepo" al celular: ya dejan usarlo en las aulas bonaerenses8

Levantan la prohibición y los chicos de Provincia ya pueden usar el celular en el aula

Página 12

- Sociedad > Levantaron la veda de teléfonos celulares en las escuelas bonaerenses Saquen una hoja y un teléfono9

La provincia de Buenos Aires derogó la resolución que prohibía los dispositivos en las aulas para docentes y alumnos, y dejó a criterio de cada establecimiento la decisión de habilitar o no su uso "como recurso pedagógico didáctico".

El cronista

- Buenos Aires: derogan la prohibición de usar celulares en las aulas10

Ahora podrán utilizarse "como recurso pedagógico". El ministro de Educación, Alejandro Finocchiaro, resaltó que apunta a "un cambio de paradigma", y que es una medida complementaria de otras más amplias. La decisión de habilitarlos o no dependerá ahora de cada establecimiento.

Infobae

- Habilitaron el uso de celulares en las aulas bonaerenses 11

El gobierno de María Eugenia Vidal derogó la prohibición que regía desde 2006. "Vamos a cambiar el paradigma de cómo se enseña en el aula", dijo el ministro de Educación de la Provincia

Ámbito

- Habilitan el uso de celulares en escuelas bonaerenses 12 
De los titulares expuestos es posible señalar dos énfasis de las noticias. Por un lado, el énfasis en el discurso regulador (prohibición/autorización) y por otro, la centralidad del dispositivo "celular" para dar cuenta del cambio en política desplegada en la provincia de Buenos Aires.

Con relación a la regulación como eje discursivo de la noticia, la mayor parte de los titulares de los diarios seleccionados refiere la autorización o a dejar sin efecto la prohibición del uso de celulares en las aulas (habilitan/derogan la prohibición/autorizan/levantan la prohibición). Se trata en su mayoría de procesos materiales de influencia que requieren de un sujeto que efectúe la acción de "liberar" una restricción preexistente. Asimismo, la mayor parte de los diarios opta por un presente durativo, a excepción de Página 12 que elige un pasado simple, por lo tanto se señala que la acción ya finalizó.

En cuanto a las metáforas, Clarín utiliza el dominio económico "el cepo", es decir, la restricción para la compra de divisa extranjera, y en particular dólares, desarrollada durante el gobierno de Cristina Fernández de Kirchner para referirse a la normativa anterior vinculada con la restricción en el uso de celulares por parte de docentes y estudiantes. Es decir, hay una analogía entre la política económica y la política educativa anterior signada por la falta de libertad de circular, usar y adquirir determinados bienes.

La metáfora de la escuela es utilizada en forma recurrente por Página 12 para presentar las noticias vinculadas con educación como también con las políticas TIC "Saquen una hoja y un teléfono". Asimismo, este diario opta por mitigar la prohibición a través del uso del término "veda" que acota la restricción a un determinado período de tiempo.

En estos titulares podemos ver regularidades en torno a la idea de un estado regulador, una autoridad que establece claras diferencias entre aquellos dispositivos que entran a la escuela como aquellos que no; también regulan las prácticas en torno al "uso pedagógico" y no pedagógico que puede tener la manipulación de los dispositivos digitales.

También es posible observar diferencias en las formas de enunciar. Esas divergencias se vinculan con las distancias analizadas en la prensa en relación con Conectar Igualdad. Así, comunicar la política es hacer política de la comunicación.

Con respecto a la jerarquización del celular por sobre el resto de los dispositivos puede obedecer a diversos factores. En términos de reproducción de la normativa, la resolución 778/16 establece la derogación de la resolución 1728/06 que prohibía utilizar teléfonos celulares por parte de docentes y alumnos13. En relación con la ubicuidad de estas tecnologías, esta situación puede responder a la proliferación de teléfonos celulares en la población. Para el año 2015, según la Encuesta Nacional sobre Acceso y Uso de Tecnologías de la Información y la Comunicación (ENTIC) la telefonía móvil es la tecnología con mayor presencia en la población después de la televisión; y un 78,2\% de la población urbana de 10 y más años usó telefonía móvil. Por último, en términos de artefacto cultural, "la necesidad de estar permanentemente localizable se debe a la necesidad de extender el anclaje doméstico y familiar en el espacio público como una forma de contrarrestar la incertidumbre" (Winocur, 2009, pp. 35). 
Las políticas de introducción de TIC en el sistema educativo se encuentran fuertemente mediatizadas. Analizar las representaciones que sobre ellas circulan en la esfera pública constituye un objetivo significativo para el logro de políticas democráticas.

Al analizar los titulares de la prensa escrita respecto del uso de dispositivos digitales del hogar en las escuelas bonaerenses en la escuela observamos una continuidad en las líneas editoriales marcadas por las diferencias en torno al gobierno nacional y a un protagonismo significativo del celular como políticas TIC en el sistema educativo. El dispositivo digital como política educativa constituye una sinécdoque que metonímicamente oculta la complejidad de la política pública y de su implementación en el aula.

Asimismo, los discursos de la esfera pública acerca de las políticas TIC acarrean modelos sociales, ubican a la autoridad, a las escuelas y a la ciudadanía en determinadas posiciones. En este sentido, constituyen una instancia de mediación de la política educativa en su conjunto.

Notas

1. Coordinado por Eva Da Porta y desarrollado en el marco del proyecto "Privatization in Education Research Initiative (Open Education Society - Campaña Latinoamericana por el Derecho a la Educación)”.

2. Asociaciones de padres de escuelas públicas que obtienen para complementar el financiamiento estatal.

3. http://one.laptop.org

4. BYOD (Bring Your Own Device) o BYOT (Bring Your Own Technology) para sus siglas en inglés.

5. http://www.lanacion.com.ar/1951139-levantan-la-prohibicion-de-usar-celulares-enlas-aulas-bonaerenses

6. http://www.lanacion.com.ar/1951006-autorizan-el-uso-de-telefonos-celulares-en-lasescuelas-bonaerenses

7. http://www.lanacion.com.ar/1951006-autorizan-el-uso-de-telefonos-celulares-en-lasescuelas-bonaerenses

8. https://www.clarin.com/sociedad/fin-celular-usarlo-aulas-bonaerenses_0_Hy8IVnelg. html

9. https://www.pagina12.com.ar/diario/sociedad/3-312862-2016-10-28.html

10. http://www.cronista.com/informaciongral/Buenos-Aires-derogan-la-prohibicion-deusar-celulares-en-las-aulas20161027-0126.html

11. http://www.infobae.com/sociedad/2016/10/28/habilitaron-el-uso-de-celulares-en-lasaulas-bonaerenses/

12. http://www.ambito.com/860421-habilitan-el-uso-de-celulares-en-escuelas-bonaerenses

13. Art. 1. Derógase la Resolución $1728 / 06$ y toda otra en tanto se oponga a la presente." Art. 1. Prohibir en todo el Sistema Educativo de la Provincia de Buenos Aires la utilización de teléfonos celulares al personal docente y a los alumnos que sean portadores y/o usuarios de los mismos, dentro del ámbito escolar y en horario de clase". 
Becerra, M. y Beltrán, R. (Comp.) (2014). Medios y TIC en la Argentina. Estudio sobre adopción de tecnologías de la información en medios de comunicación. UBACyT, Universidad de Buenos Aires. Disponible en:

https://martinbecerra.files.wordpress. com/2014/12/medios-y-tic-en-la-argentina-ubacyt-becerra-beltran-2014.pdf

Becerra, M. y Mastrini, G. (2009). Los dueños de la palabra: acceso, estructura y concentración de los medios en la América latina del siglo XXI. Buenos Aires: Prometeo.

Boczkowski, P. y Mitchelstein, E. (2015). La brecha de las noticias. La divergencia entre las preferencias informativas de los medios y el público. Buenos Aires: Manantial.

Da Porta, E., Cianci, M. (2016). Mediatización de los procesos de privatización de -y en- la educación en América Latina y el Caribe: un estudio de los discursos mediáticos. Educação \& Sociedade, Enero-Marzo, pp. $35-54$.

Da Porta, E. (Comp.) (2015) Las significaciones de las TIC en Educación. Políticas, proyectos, prácticas. Córdoba: Ferreyra editor.

Da Porta, E. (2015, 29 de octubre). Entrevista a Eva Da Porta. Recuperado de http:// privatizacion.campanaderechoeducacion.org/entrevista-en-la-prensa-hegemonica-laeducacion-aparecevinculada-a-la-escuela-publica-como-caos-y-a-la-escuela-privadacomo-calidad/

Dussel, I. (2016). Perspectivas, tensiones y límites en la evaluación de las políticas Uno a Uno en América Latina. En: Benitez Langhi, S y Winocur, R. (Coord.) Inclusión digital. Una mirada crítica sobre la evaluación del Modelo Uno a Uno en Latinoamérica. Buenos Aires: Teseo.

Cuban, L (2001). Oversold and underused. Computers in Classromm. Cambridge, Massachusetts: Harvard University Press.

INTEF (Instituto Nacional de Tecnologías Educativas y de Formación del Profesorado) (2016). Departamento de Proyectos Europeos. Diseñando el aula del futuro. Bring your own device (BYOD): una guía para directores y docentes. Recuperado de: http://blog.educalab. es/intef/wpcontent/uploads/sites/4/2016/02/Informe_resumen_BYOD_EUN_Enero_2016_INTEF.pdf

Lakoff, G. (2007) No pienses en un elefante. Lenguaje y debate político. Madrid: Editorial complutense.

Lakoff, G. y Johnson, M. (1995). Las metáforas de la vida cotidiana. Madrid: Cátedra.

Landau, M. (2015). Las TIC en la escuela en los discursos de la prensa escrita. El caso Conectar igualdad. En Pérez Fernández, S. y Armand, S. Pixelaciones. Buenos Aires: Ciccus.

Sunkel, G., Trucco, D. y Espejo, A. (2014). La integración de las tecnologías digitales en las escuelas de América Latina y el Caribe. Una mirada multidimensional. Comisión Econó- mica para América Latina y el Caribe 
(CEPAL) Santiago de Chile. Disponible en: http://

repositorio.cepal.org/bitstream/handle/11362/36739/S20131120_es.pdf

Schuliaquer, I. (2017). ¿Quién domina la escena? La relación entre los gobiernos del Frente Amplio y las empresas mediáticas en Uruguay (2005'2015) Mitchelstein, E. y Boczkowski, P. (Compiladores) Titulares, Hashtags y Videojuegos. La comunicación en la era digital. Buenos Aires: Manantial.

Thomas, S. (2004). Reconfiguring the public sphere: Implications for analyses of educational policy. British Journal of Educational Studies 52(3):228 - 248.

Verón, E (1983). Construir el acontecimiento. Barcelona: Gedisa.

Winocur, R. y Sánchez Vilella, R. (s/d). La experiencia de apropiación de las computadoras XO en las familias y comunidades beneficiarias del Plan CEIBAL. Informe ejecutivo. Disponible en:

file://C:/Users/Ivana/Downloads/Evaluacion-cualitativa-de-las-experiencias-deapropiaci\%C3\%B3n-de-lascomputadoras-INFORME-EJECUTIVO.pdf

Winocur, R (2009). Robinson Crusoe ya tiene celular. La conexión como espacio de control de la incertidumbre. México: Siglo XXI.

Abstract: This paper analyzes the speeches of the written press on the issue of the integration of information and communication technologies (ICT) in the educational system at the time of launching educational policy initiatives related to the theme in the last decade in the Argentina. In this case, we will focus on the study of the owners of the written press linked to the modification of the norms that regulate the use of cellular and other digital devices owned by students in the school environment in the province of Buenos Aires.

Key words: Newspaper - ICT - Cell phones - Public Politics

Resumo: Este artigo analisa os discursos da imprensa escrita sobre o problema da integração das tecnologias da informação e da comunicação (TIC) no sistema educacional, no momento do lançamento de iniciativas de políticas educacionais ligadas ao tema, na última década na Argentina. Neste caso, estudaremos as manchetes da imprensa escrita ligada a modificação das regras que regulam o uso de telefones celulares e outros dispositivos digitais de propriedade dos estudantes no meio escolar na província de Buenos Aires.

Palavras chave: imprensa escrita - TIC - telefone celular - políticas públicas.

[Las traducciones de los abstracts al inglés y portugués fueron supervisadas por el autor de cada artículo]

Los discursos sobre tecnologías y educación en la esfera pública fue publicado de la página 81 a página93 en Cuadernos del Centro de Estudios de Diseño y Comunicación №72 